\section{Visual tilt normalization: The method of kinesthetic matching*}

\author{
WILLIAM B. TEMPLETON \\ York University, Downsview, Ontario, Canada
}

Changes in the apparent tilt of a visual line over time were tracked by matching the orientation of a rod held in the hands. The degree of overestimation of 20-deg tilts diminished over time by about 2 deg. This occurred even though the adaptation line was exposed intermittently with a $1: 3$ on/off ratio.

Two major theories have traditionally been claimed to account for the aftereffects of seen tilt and curvature, i.e., the fact that, after inspection of a tilted (curved) line, a vertical (straight) line typically appears tilted (curved) away from the inspected figure. Gibson and Radner (1937) considered both effects to be the result of normalization in an oppositional scale, i.e., a stimulus dimension that has a centrally located norm or neutral point, with respect to which judgments of other scale values are made. When a stimulus that deviates from the norm is inspected for a period, it comes to appear more like the norm and, in the process, produces an alteration over the whole scale in the correspondence between physical values and reported magnitudes. Stimuli actually at the norm are now apparently deviated away from the location of the inspected figure.

Köhler and Wallach (1944), on the other hand, considered the visual aftereffects as special cases of a broader class of contour/repulsion phenomena dependent on a mechanism that they called "satiation." On this theory, the norm (verticality or straightness) would have no special significance and the aftereffects would depend simply on the separation of inspection and test figures rather than on their absolute location on the scale.

Howard and Templeton (1966) listed a number of critical differences between the theories, one of which hinges on the existence of normalization, a cornerstone of one theory and a phenomenon difficult, if not impossible, to explain by the other. However, although normalization is often reported by $\mathrm{Ss}$ (e.g., Gibson \& Radner, 1937; Morant \& Mistovich, 1960), it is extremely difficult to demonstrate rigorously and quantitatively. The obvious method of asking the $S$ how many degrees a single

*This research was supported by NRC Grant N. A0299. line appears to be tilted, before and pointless, because judgments away from the main anchoring points of the because freely chosen response categories tend to change in 5-deg steps at best, which is hardly sufficient to detect a change of $2 \mathrm{deg}$. In addition, later judgments are no doubt influenced by earlier ones when only one line is used in the experiment. The alternative method is to embed the inspection line in a series of test lines and to ask for magnitude estimates of the whole series. In this case, one cannot be sure that the presence of the other test lines does not contaminate the primary effect.

A second procedure is to ask for parallelism judgments of a comparison figure located in a region of the field thought to be unaffected by the tilt about the degree of transfer of the effect, this must be a perilous procedure. Prentice and Beardslee (1950) exposed a 3 -in. inspection figure at $10 \mathrm{deg}$ from the vertical on one side of the fixation point, and, subsequently, a similar test figure the same distance on the other side. The $S$ reported whether the test figure appeared more or less tilted than the inspection figure had been. The reported normalization was about $2 \mathrm{deg}$, and the fact that this was not altered by making the square frame twice as large, nor, indeed, by dispensing. with it altogether, was claimed as evidence against contamination by figural aftereffects. Nor was there any effect of a parallelogram frame with its vertical sides parallel with the test and inspection lines - a condition in which supposedly no satiation effects would be predicted.

But Heinemann and Marill (1954) argued that figural aftereffects could be differentially operative, even with a parallelogram frame, since the density of satiation is greater within acute angles than within obtuse ones. They themselves repeated the experiment, using various tilt combinations of lines and frame, and found only a satiation after a period of inspection, is scale are relatively imprecise and adaptation. In view of the uncertainty effect-the inspection lines appeared to align themselves with the frame. Held (1963) used a similar procedure involving test inspection and comparison figures on opposite sides of the fixation point and reported a normalization effect. But these techniques can detect only that portion of the effect that does not transfer over the distance between the two figures. Morant and Mikaelian (1960) reported a $66 \%$ transfer of a tilt aftereffect across $7 \mathrm{deg}$ of visual angle.

Coren and Festinger (1967) argued that a progressive change in perception over time, which could properly be called "adaptation," should involve an increase in the veridicality of perception. Using a technique of matching widths and heights by means of straight comparison lines that were presumed not to influence the process under study, they demonstrated a diminution in the apparent curvature of a curved line (normalization); moreover, the change was from an initial overestimation of curvature towards veridicality. The change was attributed to a decrease in the apparent three-dimensional appearance of the stimulus.

The concern of the present study is with tilt rather than with curvature, and, in this case, it is difficult to see how an apparent depth explanation could be relevant. Nor, for obvious reasons, is the technique of measuring apparent height and width by means of comparison lines applicable. However, it is hoped, by the use of a kinesthetic matching task, to establish the existence of visual tilt normalization, and Coren and Festinger's suggestion that this should involve increasing rather than decreasing veridicality would seem a reasonable expectation.

In the experiment, $S$ s adjusted a rod to match the apparent orientation of a visible tilted line, first with only brief exposures of the target interspersed with longer periods of darkness, then with continuous exposure of the line.

\section{METHOD \\ Apparatus}

A hollow metal cylinder, 18 in. long and 8 in. in diam, was mounted with its long axis horizontal and about head high for a seated $S$. $S$ viewed the targets binocularly through a viewing mask mounted on one end of the cylinder. The target was a 6-in. plastic rod, painted black except for a 1/16-in. groove milled along its length. The rod was illuminated by a pea-bulb fixed at each end, so that only the milled groove was visible as a thin line of light. It was mounted in S's frontal-parallel plane at a distance of about 12 in. and could be rotated 
Table 1

Analysis of $V$ ariance of Overestimation of Tilt

\begin{tabular}{|c|c|c|c|}
\hline Source & df & $\begin{array}{c}\text { Mean } \\
\text { Square }\end{array}$ & $\mathbf{F}$ \\
\hline Ss & 7 & 11443 & \\
\hline Blocks & $\mathbf{2}$ & 6493 & $17.1 *$ \\
\hline Blocks by Ss & 14 & $\mathbf{3 8 0}$ & \\
\hline Targets & $\mathbf{3}$ & 4969 & n.s. \\
\hline Targets by Ss & 21 & 9430 & \\
\hline Starting Position & 1 & 190385 & $5.9 \dagger$ \\
\hline Starting Position by Ss & 7 & 32564 & \\
\hline Blocks by Targets & 6 & 116 & n.s. \\
\hline Blocks by Targets by Ss & 42 & $\mathbf{3 5 3}$ & \\
\hline Blocks by SPs & 2 & 366 & n.s. \\
\hline Blocks by SPs by Ss & 14 & 450 & \\
\hline Targets by SPs & $\mathbf{3}$ & 1640 & n.s. \\
\hline Targets by SPs by Ss & 21 & 2886 & \\
\hline Blocks by Targets by SPs & 6 & 263 & n.s. \\
\hline Blocks by Targets by SPs by Ss & 42 & 181 & \\
\hline Total & 191 & & \\
\hline
\end{tabular}

about its center in that plane by means of a spindle that was supported in a bearing in the center of the far wall of the cylinder. The protruding end of the spindle carried a pointer that rotated on a degree scale mounted on the outside of the end wall. The middle $1 / 4 \mathrm{in}$. of the groove was occluded to provide a fixation point.

A large bearing surrounded the cylinder at the same distance as the target, and this carried two $8 \times 1 / 4$ in. square metal rods, which formed the extensions of a diameter of the cylinder. $S$ could grasp the outer ends of these rods, one with each hand, and comfortably rotate them in the frontal plane through more than $180 \mathrm{deg}$, i.e., from beyond vertical with right hand on top to beyond vertical with left hand on top. The cylinder was supported in such a manner that $S$ encountered no obstruction during this rotation.

It thus appeared to $\mathbf{S}$ that he was grasping the two ends of a single unseen rod that rotated in the same plane and about the same center as the visible target line. The angular position of the rods could be read off the scale at the back of the cylinder to the nearest $1 / 2 \mathrm{deg}$.

\section{Procedure}

Each $S$ made 30 adjustments at 8-sec intervals under each of eight conditions. For each adjustment, $S$ grasped the rod at both ends and rotated it until it appeared aligned with the visible target line. He then released the rod and dropped his hands to his lap until the next trial was signaled. For the first 10 trials (Block 1) under each condition, the target was visible for only $2 \mathrm{sec}$ and was off for the remaining 6 sec. For the most part, Ss completed their adjustments during the 2-sec on period. On the remaining 20 trials (Blocks 2 and 3), the target was continuously visible and an adjustment was signaled every $8 \mathrm{sec}$. While the target was visible, $\mathrm{S}$ was required to fixate its dark center.

Taking horizontal as $\mathbf{0} \mathrm{deg}$, the four target locations were $20,70,110$, and $160 \mathrm{deg}$, i.e., $20 \mathrm{deg}$ on either side of vertical and horizontal. Throughout any one condition, the starting position of the rod was the same, either vertical or horizontal. Half the Ss were given the four target locations with the vertical starting position first, then the four target locations in the same order with the horizontal starting position. The other half used the horizontal starting position first. Half of each of these groups was given the target locations in the order 20,70 , 110 , and $160 \mathrm{deg}$, the other half having this order reversed. Conditions were separated by about $5 \mathrm{~min}$, during which $\mathrm{S}$ relaxed with the room lights on. Because of the travel limitations imposed by the cylinder, starting positions were not symmetrical about any one target location, but this factor was balanced across target locations, e.g., although the clockwise approach to $20 \mathrm{deg}$ was shorter than the counterclockwise approach, the reverse was true of the $70-\mathrm{deg}$ locations. Similarly, target locations near the vertical and horizontal are comparable, since one of each class had a short clockwise approach and a long counterclockwise approach, whereas the other had the reverse.

\section{Subjects}

There were eight $\mathrm{Ss}$, five males and three females, all aged between 18 and 25 years and all naive as to the purpose of the experiment.

\section{RESULTS}

The data to be analyzed are the means of the 10 settings made by each $S$ in each of the three blocks in each of the two directions of adjustment to each of the four target locations. For simplicity of interpretation, the data were manipulated so that normalization would appear as a main effect rather than as an interaction. This was achieved by signing each setting as a deviation away from (positive) or towards (negative) the main axis closest to the target location. Normalization would thus produce a negative shift from Block 1 to Blocks 2 and 3 for all four target locations. Similarly, it was arranged that any systematic effect of starting position should appear as a main effect by classifying the starting position according to whether it was the main axis nearer to or farther from the target location. Thus, for the $70-$ and $110-\mathrm{deg}$ targets, the vertical was the "near starting position" and the horizontal the "far starting position," while for the 20- and 160-deg targets, the roles of the axes were reversed. The analysis of variance is shown in Table 1.

The only significant effects are blocks and starting positions. Both of these remain significant when Geisser and Greenhouse's conservative $F$ test is substituted. These effects are displayed in Table 2. The overall means for Blocks 1,2 , and 3 are 3.1 , 1.3 , and $1.3 \mathrm{deg}$, respectively, so the significance is clearly explained by the negative shift from Block 1 to Block 2. This pattern is consistent for all four targets as shown by the small mean square for Blocks by Targets. There is a tendency for the shift between Block 2 and Block 3 to be positive when the near starting position is used and negative when the far starting position is used, but this is not

Table 2

Mean Overestimation of Tilt (Degrees) for Four Targets and Near and Far Starting Positions Over Three Trial Blocks

\begin{tabular}{|c|c|c|c|c|c|c|c|c|c|}
\hline \multirow[b]{3}{*}{ Blocks } & \multirow{2}{*}{\multicolumn{2}{|c|}{$\frac{\text { Target } 20}{\text { Startin }}$}} & \multirow{2}{*}{\multicolumn{2}{|c|}{ Target 70}} & \multirow{2}{*}{\multicolumn{2}{|c|}{$\frac{\text { Target } 110}{\text { Starting }}$}} & \multicolumn{2}{|c|}{ Target 160} & \multirow[b]{3}{*}{ Mean } \\
\hline & & & & & & & \multicolumn{2}{|c|}{ Positions } & \\
\hline & Near & Far & Near & Far & Near & Far & Near & Far & \\
\hline $\begin{array}{l}\mathbf{1} \\
\mathbf{2} \\
\mathbf{3}\end{array}$ & $\begin{array}{l}-1.1 \\
-1.8 \\
-2.2\end{array}$ & $\begin{array}{l}4.9 \\
2.6 \\
2.7\end{array}$ & $\begin{array}{r}1.5 \\
-1.2 \\
-1.9\end{array}$ & $\begin{array}{l}7.3 \\
6.2 \\
6.3\end{array}$ & $\begin{array}{r}1.0 \\
-0.6 \\
-1.3\end{array}$ & $\begin{array}{l}6.6 \\
4.5 \\
5.0\end{array}$ & $\begin{array}{l}-1.4 \\
-3.0 \\
-\mathbf{3 . 0}\end{array}$ & $\begin{array}{l}5.8 \\
4.0 \\
5.2\end{array}$ & $\begin{array}{l}3.1 \\
1.3 \\
1.3\end{array}$ \\
\hline Mean & -1.7 & 3.4 & -0.5 & 6.6 & -0.3 & 3.4 & -2.5 & 5.0 & \\
\hline
\end{tabular}




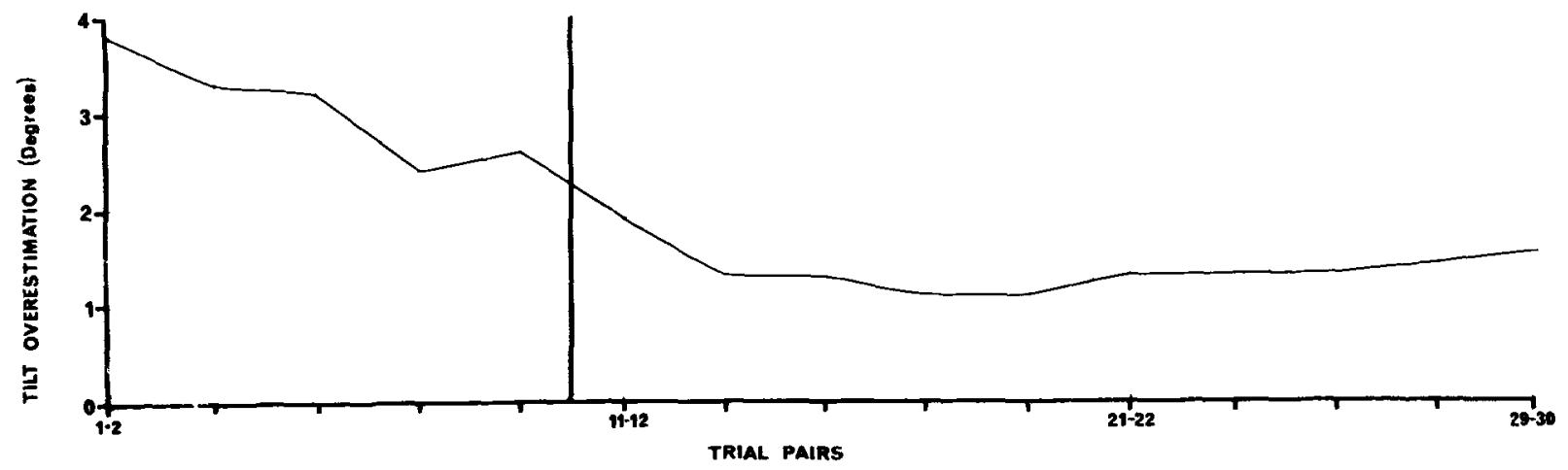

Fig. 1. Overestimation of tilt from nearest axis-means of successive pairs of trials. Vertical line indicates change of exposure conditions between Block 1 and Block 2.

significant, as shown by the small mean square for Blocks by Starting Positions.

The starting position effect is due to a tendency to undershoot, i.e., errors are relatively negative (closer to the near main axis) when the near main axis is the starting position and relatively positive when the far main axis is the starting position. This effect is consistent for all targets and all blocks.

It remains to be ascertained whether the brief stimulus exposures in Block 1 had, indeed, no net cumulative effect, in which case the significant difference would represent a homogeneous Block 1 followed by a sharp drop. Accordingly, the data are shown again in Fig. 1 as means of successive pairs-rather than tens-of trials. Since the preceding analysis shows the normalization to be the same for all target locations and starting positions, the data points in Fig. 1 are averaged over these variables. It is clear that, contrary to expectation, there is an adaptive change during Block 1 and that the transition to Block 2 is merely a continuation of this change apparently little affected by the change in exposure conditions. Indeed, about $50 \%$ of the total normalization occurs during Block 1.

\section{DISCUSSION}

The experiment would appear to demonstrate that visual lines tilted $20 \mathrm{deg}$ in either direction from the vertical or horizontal suffer an apparent shift of orientation of at least 2 deg towards the nearer main axis. This is about the magnitude usually reported for the tilt aftereffect (Gibson \& Radner, 1937; Howard \& Templeton, 1964; Mikaelian \& Held, 1964) and might, therefore, be taken as support for Gibson's original view that the latter effect results from normalization, in contrast to the two-factor theories of the tilt aftereffect that invoke normalization and a figural aftereffect (Morant \& Harris, 1965; Templeton, Howard, \& Easting, 1966). However, this inference is probably unwarranted, since the aftereffect has usually been measured by the method of adjustment, which allows time for some decay and therefore leads to underestimation of the effect.

The actual normalization consists of a decrease in the amount of overestimation of the deviation of a visual line from the nearer main axis from 3-4 deg to 1-2 deg. This conclusion is based on the assumption that there is negligible constant error in the kinesthetic system itself and, if valid, would support Coren and Festinger's results with visual curvature.

That the reported normalization is a truly visual phenomenon and not an artifact of a shift in kinesthetic judgment is strongly implied by reports of kinesthetic tilt aftereffects analogous to those in vision. This suggests that the measured normalization may actually be an underestimate, since what is measured would be the difference between the visual and possible kinesthetic effects. Other kinesthetic effects such as progressive changes in the degree of undershooting would seem to be incapable of materially affecting the results, since the direction of approach to the target was alternated.

The significant undershooting itself is presumably due either to an overestimation of current excursions in the motor-kinesthetic system or, perhaps more likely, to a visual capture effect whereby a kinesthetically sensed stimulus approaching a visual stimulus seems to have reached it before it actually has. This effect would repay further investigation.

\section{COREN REFERENCES}

OREN, S., \& FESTINGER, L. An alternative view of the "Gibson normalization effect." Perception \& Psychophysics, 1967, 2, 621-626.

GIBSON, J. J., \& RADNER, M. Adaptation, after-effect, and contrast in the perception of tilted lines. I. Quantitative studies. Journal of Experimental Psychology, 1937, 20, 453-467.

HEINEM ANN, E. G.. \& MARILL, T. Tult adaptation and figural after-effects. Journal of Experimental Psychology, $1954,48,468-472$

HELD, R. Localized normalization of tilted lines. American Journal of Psychology. $1963,76,146-148$.

HOWARD, I. P., \& TEMPLETON, W. B Visually-induced eye torsion and tilt adaptation. Vision Research, 1964, 4, 433-437.

HOWARD, I. P., \& TEMPLETON, W. B. Human spatial orientation. London: Wiley, 1966.

KÖHLER W. \& WALLACH, H. Figural after-effects: An investigation of visual processes. Proceedings of the Amexican Philosophical Society, 1944, 88, 269-357. MIKAELIAN, H., \& HELD, R. Two types of adaptation to an optically-rotated visual field. American Journal of Psychology, 1964, 77, 257-263.

MORANT, R. B., \& HARRIS, J, R. Two different after-effects of exposure to visual tilts. American Journal of Psychology, 1965, 78, 218-226.

MORANT, R. B., \& MIKAELIAN, H. H. Inter-field tilt after-effects. Perceptual \& Motor Skills, 1960, 10, 95-98.

MORANT R, B. \& MISTOVICH, M. Tilt after-effects between the vertical and horizontal axes. Perceptual \& Motor Skills, 1960, 10, 75-81.

PRENTICE, w. C. H., \& BEARDSLEE, D C. Visual "normalization" near the vertical and horizontal. Journal of Experimental Psychology, 1950, 40, Experiment

TEMPLETON, W. B., HOWARD, I. P., \& EASTING, G. Satiation and the tilt after-effect. American Journal of Psychology, 1965, 78, 656-659.

(Received for publication March 1, 1972; revision received $M a y$ 7, 1972.) 